\title{
MULTIPLE SOLUTIONS FOR NEUMANN SYSTEMS IN AN ORLICZ-SOBOLEV SPACE SETTING
}

\author{
GHASEM A. AFROUZI, JOHN R. GRAEF, AND SAEID SHOKOOH
}

Received 05 December, 2015

\begin{abstract}
In this paper, the authors improve some results on the existence of at least three weak solutions for non-homogeneous systems. The proof of the main result relies on a recent variational principle due to Ricceri.
\end{abstract}

2010 Mathematics Subject Classification: 34B37; 35J60; 35J70; 46N20; 58E05

Keywords: three solutions, non-homogeneous differential operator, Orlicz-Sobolev space

\section{INTRODUCTION}

This paper is motivated by phenomena that are described by non-homogeneous Neumann double eigenvalue systems of the type

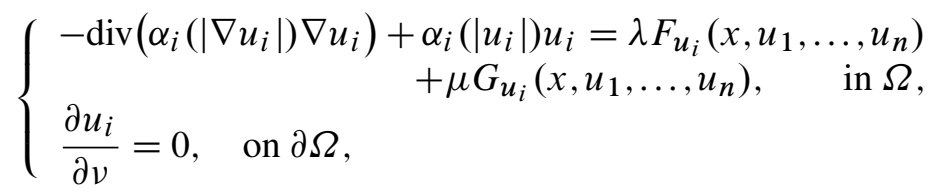

for $1 \leq i \leq n$, where $\Omega$ is a bounded domain in $\mathbb{R}^{N}(N \geq 3)$ with a smooth boundary $\partial \Omega, v$ is the outward unit normal to $\partial \Omega, \lambda$ and $\mu$ are positive parameters, $F, G: \Omega \times$ $\mathbb{R}^{n} \rightarrow \mathbb{R}$ are measurable functions with respect to $x \in \Omega$ for every $\left(t_{1}, \ldots, t_{n}\right) \in \mathbb{R}^{n}$ and are $C^{1}$ with respect to $\left(t_{1}, \ldots, t_{n}\right) \in \mathbb{R}^{n}$ for a.e. $x \in \Omega$. Here, $F_{u_{i}}$ and $G_{u_{i}}$ denote the partial derivative of $F$ and $G$ with respect to $u_{i}$, respectively, and the functions $\alpha_{i}(t): \mathbb{R} \rightarrow \mathbb{R}$ for $1 \leq i \leq n$ will be specified later.

For any function $H: \Omega \times \mathbb{R}^{n} \rightarrow \mathbb{R}$, we denote by (H0) the following assumption on $H$ :

(H0) $H$ is measurable with respect to $x \in \Omega$ for every $\left(t_{1}, \ldots, t_{n}\right) \in \mathbb{R}^{n}$, and for every $M>0$ and for every $1 \leq i \leq n$,

$$
\sup _{\left|\left(t_{1}, \ldots, t_{n}\right)\right| \leq M}\left|H_{u_{i}}\left(x, t_{1}, \ldots, t_{n}\right)\right| \in L^{1}(\Omega) .
$$

Our approach in this paper relies on variational methods in Orlicz-Sobolev spaces. Such spaces originated with Nakano [26] and were further developed by Musielak 
and Orlicz [25]. Many properties of Sobolev spaces have been extended to OrliczSobolev spaces, mainly by Dankert [11], Donaldson and Trudinger [14], and O'Neill [27] (see also [1] for an excellent account of those works). Orlicz-Sobolev spaces have been used in the last two decades to model various phenomena. These kind of spaces play a significant role in many fields of mathematics, such as approximation theory, partial differential equations, calculus of variations, nonlinear potential theory, quasiconformal mappings, differential geometry, geometric function theory, and probability theory. Due to this, several authors have widely studied the existence of solutions for eigenvalue problems involving non-homogeneous operators (see, for example, [2,3,5-10, 15, 17, 18, 20-22,33]).

Notice that if $i=1$ and $\mu=0$, then problem (1.1) becomes

$$
\left\{\begin{array}{l}
-\operatorname{div}(\alpha(|\nabla u|) \nabla u)+\alpha(|u|) u=\lambda f(x, u), \text { in } \Omega \\
\frac{\partial u}{\partial v}=0, \quad \text { on } \partial \Omega .
\end{array}\right.
$$

Bonanno, Molica Bisci, and Rădulescu [5] used a recent critical points result in order to prove the existence of an open interval of positive eigenvalues for which the problem (1.2) admits at least three weak solutions. The same authors in [6] established an open interval of positive parameters for which the problem (1.2) admits infinitely many weak solutions that strongly converges to zero.

In [8] Cammaroto and Vilasi proved the existence of three weak solutions to the nonhomogeneous boundary value problem:

$$
\left\{\begin{array}{l}
-\operatorname{div}(\alpha(|\nabla u|) \nabla u)=\lambda f(x, u)+\mu g(x, u), \text { in } \Omega, \\
u=0, \quad \text { on } \partial \Omega .
\end{array}\right.
$$

Note that if we take $\alpha(t)=|t|^{p-2}(p>1)$ in problems (1.2) and (1.3), the operator on the left hand side reduces to the classical $p$-Laplacian; these types of problems have been widely investigated and a large variety of existence results can be found in the literature.

The classical three critical point theorem of Pucci and Serrin [28,29] asserts that if $h: Y \rightarrow \mathbb{R}$ is of class $C^{1}$ ( $Y$ is a Banach space), satisfies the Palais-Smale condition, and has two local minima, then $h$ has a third critical point. The general variational principle of Ricceri $[31,32]$ extends the Pucci-Serrin theorem and provides alternatives for the multiplicity of critical points of certain functionals depending on a parameter. We refer to [19] and the monograph of Graef and Kong [16] for several applications of Ricceri's variational principles.

In this paper, motivated by the above facts and the recent paper of Molica Bisci and Rădulescu [23], under appropriate conditions on $F$ and $G$, we present sufficient conditions for the system (1.1) to possesses three weak solutions in an Orlicz-Sobolev space. 
Our paper is organized as follows. In Section 2, some preliminaries and the abstract Orlicz-Sobolev space settings are presented. In Section 3, we discuss the existence of three weak solutions for the system (1.1). We also point out special cases of the results and we illustrate the results by presenting an example.

\section{FunCtional SETting}

We now recall the following three critical points theorem that follows from a combination of [4, Theorem 3.6] and [32, Theorem 1].

Theorem 1. Let $X$ be a reflexive real Banach space, $J: X \rightarrow \mathbb{R}$ be a sequentially weakly lower semicontinuous and continuously Gâteaux differentiable functional that is bounded on bounded subsets of $X$ and whose Gâteaux derivative admits a continuous inverse on the dual space $X^{*}$, and let $I: X \rightarrow \mathbb{R}$ be a continuously Gâteaux differentiable functional whose Gâteaux derivative is compact and satisfies

$$
J(0)=I(0)=0 .
$$

Assume that there exist $r>0$ and $\bar{x} \in X$ with $r<J(\bar{x})$ such that

(j) $\sup _{J(x) \leq r} I(x)<r I(\bar{x}) / J(\bar{x})$,

(jj) for each $\lambda$ in

$$
\Lambda_{r}:=\left(\frac{J(\bar{x})}{I(\bar{x})}, \frac{r}{\sup _{J(x) \leq r} I(x)}\right),
$$

the functional $J-\lambda I$ is coercive.

Then, for each compact interval $[a, b] \in \Lambda_{r}$, there exists $\rho>0$ with the following property: for every $\lambda \in[a, b]$ and every $C^{1}$ functional $\Gamma: X \rightarrow \mathbb{R}$ with compact derivative, there exists $\delta>0$ such that, for each $\mu \in[0, \delta]$, the equation $J^{\prime}(x)-$ $\lambda I^{\prime}(x)-\mu \Gamma^{\prime}(x)=0$ has at least three solutions in $X$ whose norms are less than $\rho$.

In order to study the problem (1.1), let us introduce the function spaces settings to be used. We will give just a brief review of some basic concepts and facts of the theory of Orlicz-Sobolev spaces that will be useful in what follows; for more details we refer the reader to Adams [1], Diening [12], Musielak [24], and Rao and Ren [30].

We begin by recalling some facts from the theory of Orlicz-Sobolev spaces that will be used in the present paper. Suppose that the function $\alpha: \mathbb{R} \rightarrow \mathbb{R}$ is such that the mapping $\varphi: \mathbb{R} \rightarrow \mathbb{R}$ defined by

$$
\varphi(t)= \begin{cases}\alpha(|t|) t, & \text { for } t \neq 0, \\ 0, & \text { for } t=0,\end{cases}
$$

is an odd and strictly increasing homeomorphism from $\mathbb{R}$ onto $\mathbb{R}$. Consider the functions

$$
\Phi(t)=\int_{0}^{t} \varphi(s) d s \quad \text { and } \quad \Phi^{\star}(t)=\int_{0}^{t} \varphi^{-1}(s) d s \quad \text { for } \quad t \in \mathbb{R} .
$$


We observe that $\Phi$ is a Young's function, that is, $\Phi(0)=0, \Phi$ is convex, and

$$
\lim _{t \rightarrow \infty} \Phi(t)=+\infty
$$

Furthermore, since $\Phi(t)=0$ if and only if $t=0$,

$$
\lim _{t \rightarrow 0} \frac{\Phi(t)}{t}=0, \quad \text { and } \quad \lim _{t \rightarrow \infty} \frac{\Phi(t)}{t}=+\infty,
$$

$\Phi$ is called an $N$-function. The function $\Phi^{\star}$ is called the complementary function of $\Phi$ and satisfies

$$
\Phi^{\star}(t)=\sup \{s t-\Phi(s): s \geq 0\} \text { for all } t \geq 0 .
$$

We note that $\Phi^{\star}$ is also an $N$-function and the following Young's inequality holds:

$$
s t \leq \Phi(s)+\Phi^{\star}(t), \quad \text { for all } s, t \geq 0 .
$$

Assume that $\Phi$ satisfies the structural hypotheses

$$
1<\liminf _{t \rightarrow \infty} \frac{t \varphi(t)}{\Phi(t)} \leq \varphi^{+}:=\sup _{t>0} \frac{t \varphi(t)}{\Phi(t)}<\infty
$$

and

$$
N<\varphi^{-}:=\inf _{t>0} \frac{t \varphi(t)}{\Phi(t)}<\liminf _{t \rightarrow \infty} \frac{\log (\Phi(t))}{\log (t)} .
$$

We also assume that the function

$$
t \rightarrow \Phi(\sqrt{t}) \text { for } t \in[0, \infty)
$$

is convex. To illustrate these notions, we have the following example.

Example 1. Let $p>N+1$. Define

$$
\varphi(t)=\frac{|t|^{p-2}}{\log (1+|t|)} t \quad \text { for } \quad t \neq 0, \quad \varphi(0)=0,
$$

and

$$
\Phi(t)=\int_{0}^{t} \varphi(s) d s
$$

By [9, Example 3, p. 243], we have

$$
\varphi^{-}=p-1<\varphi^{+}=p=\liminf _{t \rightarrow \infty} \frac{\log (\Phi(t))}{\log (t)} .
$$

Thus, conditions $(\Phi 0)$ and $(\Phi 1)$ are satisfied. Furthermore, by a direct computation, we see that the function $t \mapsto \Phi(\sqrt{t})$ for $t \in[0, \infty)$ is convex, so $(\Phi 2)$ is also satisfied. 
The Orlicz space $L_{\Phi}(\Omega)$ defined by the $N$-function $\Phi$ (see for instance [1] and [13]) is the space of measurable functions $u: \Omega \rightarrow \mathbb{R}$ such that

$$
\|u\|_{L_{\Phi}}:=\sup \left\{\left|\int_{\Omega} u(x) v(x) d x\right|: \int_{\Omega} \Phi^{\star}(|v(x)|) d x \leq 1\right\}<\infty .
$$

The space $\left(L_{\Phi}(\Omega),\|\cdot\|_{L_{\Phi}}\right)$ is a Banach space whose norm is equivalent to the Luxemburg norm

$$
\|u\|_{1, \Phi}:=\inf \left\{k>0: \int_{\Omega} \Phi\left(\frac{u(x)}{k}\right) d x \leq 1\right\} .
$$

We denote by $W^{1} L_{\Phi}(\Omega)$ the corresponding Orlicz-Sobolev space for problem (1.1) defined by

$$
W^{1} L_{\Phi}(\Omega)=\left\{u \in L_{\Phi}(\Omega): \frac{\partial u}{\partial x_{j}} \in L_{\Phi}(\Omega), j=1, \ldots, N\right\} .
$$

This is a Banach space with respect to the norm

$$
\|u\|_{2, \Phi}=\||\nabla u|\|_{1, \Phi}+\|u\|_{1, \Phi}
$$

see $[1,10,15]$.

The following two lemmas can be found in [18].

Lemma 1. ([18, Lemma 2.2]) In $W^{1} L_{\Phi}(\Omega)$, the norms

$$
\begin{gathered}
\|u\|_{2, \Phi}=\||\nabla u|\|_{1, \Phi}+\|u\|_{1, \Phi}, \\
\|u\|_{3, \Phi}=\max \left\{\||\nabla u|\|_{1, \Phi},\|u\|_{1, \Phi}\right\},
\end{gathered}
$$

and

$$
\|u\|_{\Phi}=\inf \left\{\mu>0 ; \int_{\Omega}\left[\Phi\left(\frac{|u(x)|}{\mu}\right)+\Phi\left(\frac{|\nabla u(x)|}{\mu}\right)\right] d x \leq 1\right\}
$$

are equivalent. More precisely, for every $u \in W^{1} L_{\Phi}(\Omega)$ we have

$$
\|u\|_{\Phi} \leq 2\|u\|_{3, \Phi} \leq 2\|u\|_{2, \Phi} \leq 4\|u\|_{\Phi} .
$$

Lemma 2. ([18, Lemma 2.3]) Let $u \in W^{1} L_{\Phi}(\Omega)$. Then:

$$
\begin{gathered}
\int_{\Omega}[\Phi(|u(x)|)+\Phi(|\nabla u(x)|)] d x \geq\|u\|_{\Phi}^{\varphi^{-}}, \quad \text { if }\|u\|_{\Phi}>1 ; \\
\int_{\Omega}[\Phi(|u(x)|)+\Phi(|\nabla u(x)|)] d x \geq\|u\|_{\Phi}^{\varphi^{+}}, \quad \text { if }\|u\|_{\Phi}<1 .
\end{gathered}
$$

These spaces generalize the usual spaces $L^{p}(\Omega)$ and $W^{1, p}(\Omega)$, in which the role played by the convex mapping $t \mapsto|t|^{p} / p$ is assumed by a more general convex function $\Phi(t)$.

Proposition 1. (See [1, p. 241 and p. 247]) The spaces $L_{\Phi}(\Omega)$ and $W^{1} L_{\Phi}(\Omega)$ are separable and reflexive Banach spaces. 
Remark 1. In view of condition $(\Phi 1)$, from [10, Lemma D.2] it follows that $W^{1} L_{\Phi}(\Omega)$ is continuously embedded in $W^{1, \varphi^{-}}(\Omega)$. On the other hand, since we assumed that $\varphi^{-}>N$, we can conclude that $W^{1, \varphi^{-}}(\Omega)$ is compactly embedded in $C^{0}(\bar{\Omega})$. Thus, we have that $W^{1} L_{\Phi}(\Omega)$ is compactly embedded in $C^{0}(\bar{\Omega})$.

Throughout this paper, the functions $\alpha_{i}: \mathbb{R} \rightarrow \mathbb{R}$ are such that the mappings $\varphi_{i}$ : $\mathbb{R} \rightarrow \mathbb{R}$ defined by

$$
\varphi_{i}(t)= \begin{cases}\alpha_{i}(|t|) t, & \text { for } t \neq 0 \\ 0, & \text { for } t=0\end{cases}
$$

for $1 \leq i \leq n$ are odd and strictly increasing homeomorphism from $\mathbb{R}$ to $\mathbb{R}$. Also, assume that the functions

$$
\Phi_{i}(t)=\int_{0}^{t} \varphi_{i}(s) d s \text { for } t \in \mathbb{R}
$$

for $1 \leq i \leq n$ satisfying the structural hypotheses $(\Phi 0),(\Phi 1)$, and $(\Phi 2)$.

We let $E$ be the Cartesian product of $n$ Orlicz-Sobolev spaces $W^{1} L_{\Phi_{i}}(\Omega)$ for $1 \leq i \leq n$, i.e., $E=\prod_{i=1}^{n} W^{1} L_{\Phi_{i}}(\Omega)$ endowed with the norm

$$
\|u\|:=\sum_{i=1}^{n}\|u\|_{\Phi_{i}}, \quad u=\left(u_{1}, \ldots, u_{n}\right) \in E .
$$

In the space $C^{0}(\bar{\Omega})$, we consider the norm $\|u\|_{\infty}:=\sup _{x \in \bar{\Omega}}|u(x)|$. Set

$$
m:=\max \left\{\sup _{u \in W^{1} L_{\Phi_{i}}(\Omega) \backslash\{0\}} \frac{\|u\|_{\infty}}{\|u\|_{\Phi_{i}}}: \text { for } 1 \leq i \leq n\right\} .
$$

Since $N<\varphi_{i}^{-}$for $1 \leq i \leq n$, from Remark 1 , the embedding $E \hookrightarrow C^{0}(\bar{\Omega}) \times \ldots \times$ $C^{0}(\bar{\Omega})$ is compact, so that $m<+\infty$.

We say that $u=\left(u_{1}, \ldots, u_{n}\right) \in E$ is a weak solution of the problem (1.1) if

$$
\begin{gathered}
\int_{\Omega} \sum_{i=1}^{n}\left(\alpha_{i}\left(\left|\nabla u_{i}(x)\right|\right) \nabla u_{i}(x) \nabla v_{i}(x)+\alpha_{i}\left(\left|u_{i}(x)\right|\right) u_{i}(x) v_{i}(x)\right) d x \\
-\lambda \int_{\Omega} \sum_{i=1}^{n} F_{u_{i}}\left(x, u_{1}(x), \ldots, u_{n}(x)\right) v_{i}(x) d x \\
-\mu \int_{\Omega} \sum_{i=1}^{n} G_{u_{i}}\left(x, u_{1}(x), \ldots, u_{n}(x)\right) v_{i}(x) d x=0
\end{gathered}
$$

for every $v=\left(v_{1}, \ldots, v_{n}\right) \in E$. 


\section{MAIN RESULTS}

We begin this section with our main existence result.

Theorem 2. Assume that $F: \Omega \times \mathbb{R}^{n} \rightarrow \mathbb{R}$ satisfies $(\mathrm{H} 0)$ and the following conditions:

(F1) there exist $d \in L^{1}(\Omega)$ and n positive constants $\beta_{i}$, with $\beta_{i}<\varphi_{i}^{-}$for $1 \leq i \leq$ $n$, such that

$$
0 \leq F\left(x, t_{1}, \ldots, t_{n}\right) \leq d(x)\left(1+\sum_{i=1}^{n}\left|t_{i}\right|^{\beta_{i}}\right)
$$

for a.e. $x \in \Omega$ and all $\left(t_{1}, \ldots, t_{n}\right) \in \mathbb{R}^{n}$;

(F2) $F(x, 0, \ldots, 0)=0$ for a.e $x \in \Omega$;

(F3) there exist $0<c_{i}<m$ and $s_{i} \in \mathbb{R}$ for $1 \leq i \leq n$ with

$$
|\Omega| \sum_{i=1}^{n} \Phi_{i}\left(\left|s_{i}\right|\right)>\min \left\{\left(\frac{c_{i}}{m}\right)^{\varphi_{i}^{+}}: 1 \leq i \leq n\right\},
$$

such that

$$
\begin{aligned}
\int_{\Omega\left|t_{1}\right| \leq c_{1}, \ldots,\left|t_{n}\right| \leq c_{n}} F\left(x, t_{1}, \ldots, t_{n}\right) d x & \sup <\frac{\min \left\{\left(\frac{c_{i}}{m}\right)^{\varphi_{i}^{+}}: 1 \leq i \leq n\right\}}{|\Omega| \sum_{i=1}^{n} \Phi_{i}\left(\left|s_{i}\right|\right)} \int_{\Omega} F\left(x, s_{1}, \ldots, s_{n}\right) d x,
\end{aligned}
$$

where $|\Omega|$ denotes the Lebesgue measure of the set $\Omega$.

Then, setting

$$
\Lambda:=\left(\frac{|\Omega| \sum_{i=1}^{n} \Phi_{i}\left(\left|s_{i}\right|\right)}{\int_{\Omega} F\left(x, s_{1}, \ldots, s_{n}\right) d x}, \frac{\min \left\{\left(\frac{c_{i}}{m}\right)^{\varphi_{i}^{+}}: 1 \leq i \leq n\right\}}{\int_{\Omega} \sup _{\left|t_{1}\right| \leq c_{1}, \ldots,\left|t_{n}\right| \leq c_{n}} F\left(x, t_{1}, \ldots, t_{n}\right) d x}\right),
$$

for each compact interval $[a, b] \subset \Lambda$, there exists $\rho>0$ with the following property: for every $\lambda \in[a, b]$ and for every function $G: \Omega \times \mathbb{R}^{n} \rightarrow \mathbb{R}$ satisfying condition (H0), there exists $\delta>0$ such that, for each $\mu \in[0, \delta]$, the system (1.1) admits at least three weak solutions in $E$ whose norms are less than $\rho$.

Proof. In order to prove the theorem, we will apply Theorem 1. For each $\left(u_{1}, \ldots, u_{n}\right) \in E$, let the functionals $J, I: E \rightarrow \mathbb{R}$ be defined by

$$
J(u):=\int_{\Omega} \sum_{i=1}^{n}\left(\Phi_{i}\left(\left|\nabla u_{i}(x)\right|\right)+\Phi_{i}\left(\left|u_{i}(x)\right|\right)\right) d x
$$


and

$$
I(u):=\int_{\Omega} F\left(x, u_{1}(x) \ldots, u_{n}(x)\right) d x .
$$

Clearly, $J$ is bounded on each bounded subset of $E$ and similar arguments as those used in [21, Lemma 4.2] imply that $J$ and $I$ are continuously Gâteaux differentiable functionals whose derivatives at the point $u=\left(u_{1}, \ldots, u_{n}\right) \in E$ are the functional $J^{\prime}(u)$ and $I^{\prime}(u)$ given by

$$
J^{\prime}(u)(v)=\int_{\Omega} \sum_{i=1}^{n}\left(\alpha_{i}\left(\left|\nabla u_{i}(x)\right|\right) \nabla u_{i}(x) \nabla v_{i}(x)+\alpha_{i}\left(\left|u_{i}(x)\right|\right) u_{i}(x) v_{i}(x)\right) d x
$$

and

$$
I^{\prime}(u)(v)=\int_{\Omega} \sum_{i=1}^{n} F_{u_{i}}\left(x, u_{1}(x) \ldots, u_{n}(x)\right) v_{i}(x) d x
$$

for every $v=\left(v_{1}, \ldots, v_{n}\right) \in E$. Moreover, since $\Phi_{i}$ for $1 \leq i \leq n$ are convex, it follows that $J$ is a convex functional, and hence it is sequentially weakly lower semicontinuous. By Lemma 2, we see that $J$ is coercive, and arguing as in the proof of [18, Lemma 3.2], we have that $J^{\prime}: E \rightarrow E^{*}$ is a uniformly monotone operator in $E$. By applying the Minty-Browder theorem (Theorem 26.A of [34]), $J^{\prime}$ admits a continuous inverse on $E^{*}$.

We claim that $I^{\prime}: E \rightarrow E^{*}$ is a compact operator. To this end, it suffices to show that $I^{\prime}$ is strongly continuous on $E$, so for fixed $\left(u_{1}, \ldots, u_{n}\right) \in E$, let $\left(u_{1 m}, \ldots, u_{n m}\right)$ $\rightarrow\left(u_{1}, \ldots, u_{n}\right)$ weakly in $E$ as $m \rightarrow+\infty$. Then we have $\left(u_{1 m}, \ldots, u_{n m}\right)$ converges uniformly to $\left(u_{1}, \ldots, u_{n}\right)$ on $\Omega$ as $m \rightarrow+\infty$ (see [34]). Since $F(x, \cdot, \ldots, \cdot)$ is $C^{1}$ in $\mathbb{R}^{n}$ for every $x \in \Omega$, the partial derivatives of $F$ are continuous in $\mathbb{R}^{n}$ for every $x \in \Omega$, so for $1 \leq i \leq n, F_{u_{i}}\left(x, u_{1 m}, \ldots, u_{n m}\right) \rightarrow F_{u_{i}}\left(x, u_{1}, \ldots, u_{n}\right)$ strongly as $m \rightarrow+\infty$. By the Lebesgue dominated convergence theorem, $I^{\prime}\left(u_{1 m}, \ldots, u_{n m}\right) \rightarrow$ $I^{\prime}\left(u_{1}, \ldots, u_{n}\right)$ strongly as $m \rightarrow+\infty$. Thus, $I^{\prime}$ is strongly continuous on $E$, which implies that $I^{\prime}$ is a compact operator by [34, Proposition 26.2]. Hence, our claim is true.

From the definitions of $J$ and $I$ and from (F2), we have

$$
J(0)=I(0)=0 .
$$

Next, set $w(x):=\left(s_{1}, \ldots, s_{n}\right)$ for any $x \in \Omega$, and

$$
r:=\min \left\{\left(\frac{c_{i}}{m}\right)^{\varphi_{i}^{+}}: 1 \leq i \leq n\right\} .
$$

Clearly, $w \in E$, and we have

$$
J(w)=|\Omega| \sum_{i=1}^{n} \Phi_{i}\left(\left|s_{i}\right|\right)>r>0 .
$$


Let $J(u) \leq r$ for $u=\left(u_{1}, \ldots, u_{n}\right) \in E$. By Lemma 2 , for $1 \leq i \leq n$, we have

$$
\|u\|_{\Phi_{i}} \leq \max \left\{r^{1 / \varphi_{i}^{-}}, r^{1 / \varphi_{i}^{+}}\right\} .
$$

Then, for $1 \leq i \leq n$,

$$
\|u\|_{\Phi_{i}} \leq \frac{c_{i}}{m}
$$

Taking into account that for each $u \in W^{1} L_{\Phi_{i}}(\Omega)$,

$$
\|u\|_{\infty} \leq m\|u\|_{\Phi_{i}}
$$

for $1 \leq i \leq n$ (see (2.1)), we obtain

$$
\left\|u_{i}\right\|_{\infty} \leq c_{i} \quad \text { for } 1 \leq i \leq n .
$$

Therefore, for every $u=\left(u_{1}, \ldots, u_{n}\right) \in E$,

$$
\begin{aligned}
\sup _{u \in J^{-1}((-\infty, r))} I(u) & =\int_{\Omega} F\left(x, u_{1}(x), \ldots, u_{n}(x)\right) d x \\
& \leq \int_{\Omega\left|t_{1}\right| \leq c_{1}, \ldots,\left|t_{n}\right| \leq c_{n}} F\left(x, t_{1}, \ldots, t_{n}\right) d x .
\end{aligned}
$$

Condition (F3) implies

$$
\begin{aligned}
\frac{\sup _{u \in J^{-1}((-\infty, r))} I(u)}{r} & \leq \frac{\int_{\Omega} \sup _{\left|t_{1}\right| \leq c_{1}, \ldots,\left|t_{n}\right| \leq c_{n}} F\left(x, t_{1}, \ldots, t_{n}\right) d x}{\min \left\{\left(\frac{c_{i}}{m}\right)^{\varphi_{i}^{+}}: 1 \leq i \leq n\right\}} \\
& <\frac{\int_{\Omega} F\left(x, s_{1}, \ldots, s_{n}\right) d x}{|\Omega| \sum_{i=1}^{n} \Phi_{i}\left(\left|s_{i}\right|\right)} \\
& =\frac{I(w)}{J(w)} .
\end{aligned}
$$

Thus, assumption ( $\mathrm{j}$ ) of Theorem 1 is satisfied.

In view of $(\mathrm{F} 1)$, the functional $J-\lambda I$ is coercive for every positive parameter $\lambda$, in particular, for every

$$
\lambda \in \Lambda \subseteq\left(\frac{J(w)}{I(w)}, \frac{r}{\sup _{J(u) \leq r} I(u)}\right),
$$

so condition (jj) of Theorem 1 holds. In addition, for every function $G: \Omega \times \mathbb{R}^{n} \rightarrow \mathbb{R}$ satisfying condition ( $\mathrm{H} 0)$, the functional

$$
\Gamma(u):=\int_{\Omega} G\left(x, u_{1}(x) \ldots, u_{n}(x)\right) d x
$$


is well defined and continuously Gâteaux differentiable on $E$ with a compact derivative given by

$$
\Gamma^{\prime}(u)(v)=\int_{\Omega} \sum_{i=1}^{n} G_{u_{i}}\left(x, u_{1}(x) \ldots, u_{n}(x)\right) v_{i}(x) d x
$$

for every $u=\left(u_{1}, \ldots, u_{n}\right), v=\left(v_{1}, \ldots, v_{n}\right) \in E$. Thus, all the conditions of Theorem 1 are satisfied. Also, note that the solutions of the equation

$$
J^{\prime}(u)-\lambda I^{\prime}(u)-\mu \Gamma^{\prime}(u)=0
$$

are exactly the weak solutions of (1.1). So, the conclusion follows from Theorem 1.

Next, we consider the special case of Theorem 2 where $F$ does not depend on $x \in \Omega$.

Theorem 3. Assume that $F: \mathbb{R}^{n} \rightarrow \mathbb{R}$ is a $C^{1}$-function satisfying

(F4) there exist $n+1$ positive constants $\eta$ and $\beta_{i}$ with $\beta_{i}<\varphi_{i}^{-}$for $1 \leq i \leq n$, such that

$$
0 \leq F\left(t_{1}, \ldots, t_{n}\right) \leq \eta\left(1+\sum_{i=1}^{n}\left|t_{i}\right|^{\beta_{i}}\right)
$$

for all $\left(t_{1}, \ldots, t_{n}\right) \in \mathbb{R}^{n}$

(F5) $F(0, \ldots, 0)=0$;

(F6) there exist $0<c_{i}<m$ and $s_{i} \in \mathbb{R}$ for $1 \leq i \leq n$ with

$$
|\Omega| \sum_{i=1}^{n} \Phi_{i}\left(\left|s_{i}\right|\right)>\min \left\{\left(\frac{c_{i}}{m}\right)^{\varphi_{i}^{+}}: 1 \leq i \leq n\right\},
$$

such that

$$
\begin{aligned}
\sup _{\left|t_{1}\right| \leq c_{1}, \ldots,\left|t_{n}\right| \leq c_{n}} F\left(t_{1}, \ldots, t_{n}\right) & \\
< & \frac{\min \left\{\left(\frac{c_{i}}{m}\right)^{\varphi_{i}^{+}}: 1 \leq i \leq n\right\}}{|\Omega| \sum_{i=1}^{n} \Phi_{i}\left(\left|s_{i}\right|\right)} F\left(s_{1}, \ldots, s_{n}\right) .
\end{aligned}
$$

Then, setting

$$
\Lambda:=\left(\frac{\sum_{i=1}^{n} \Phi_{i}\left(\left|s_{i}\right|\right)}{F\left(s_{1}, \ldots, s_{n}\right)}, \frac{\min \left\{\left(\frac{c_{i}}{m}\right)^{\varphi_{i}^{+}}: 1 \leq i \leq n\right\}}{|\Omega| \sup _{\left|t_{1}\right| \leq c_{1}, \ldots,\left|t_{n}\right| \leq c_{n}} F\left(t_{1}, \ldots, t_{n}\right)}\right),
$$


for each compact interval $[a, b] \subset \Lambda$, there exists $\rho>0$ with the following property: for every $\lambda \in[a, b]$ and for every function $G: \Omega \times \mathbb{R}^{n} \rightarrow \mathbb{R}$ satisfying condition (H0), there exists $\delta>0$ such that, for each $\mu \in[0, \delta]$, the system

$$
\left\{\begin{array}{l}
\begin{array}{r}
\operatorname{div}\left(\alpha_{i}\left(\left|\nabla u_{i}\right|\right) \nabla u_{i}\right)+\alpha_{i}\left(\left|u_{i}\right|\right) u_{i}=\lambda F_{u_{i}}\left(u_{1}, \ldots, u_{n}\right) \\
+\mu G_{u_{i}}\left(x, u_{1}, \ldots, u_{n}\right), \quad \text { in } \Omega,
\end{array} \\
\frac{\partial u_{i}}{\partial v}=0, \quad \text { on } \partial \Omega,
\end{array}\right.
$$

for $1 \leq i \leq n$, admits at least three weak solutions in $E$ whose norms are less than $\rho$.

Now, consider the problem

$$
\left\{\begin{array}{l}
-\operatorname{div}(\alpha(|\nabla u|) \nabla u)+\alpha(|u|) u=\lambda f(u)+\mu g(x, u) \quad \text { in } \Omega, \\
\frac{\partial u}{\partial v}=0 \quad \text { on } \partial \Omega
\end{array}\right.
$$

where $\lambda$ and $\mu$ are positive parameters, $f: \mathbb{R} \rightarrow \mathbb{R}$ is a continuous function, $g$ : $\Omega \times \mathbb{R} \rightarrow \mathbb{R}$ is an $L^{1}$-Carathéodory function, and $\alpha$ is as in Section 2 .

Let

$$
k:=\sup _{u \in W^{1} L_{\Phi}(\Omega) \backslash\{0\}} \frac{\|u\|_{\infty}}{\|u\|_{\Phi}}
$$

and

$$
F(t):=\int_{0}^{t} f(\xi) d \xi \text { for } t \in \mathbb{R} .
$$

The following result is a special case of Theorem 3 .

Theorem 4. Let $f: \mathbb{R} \rightarrow \mathbb{R}$ be a non-negative continuous function, and assume that the function $F$ in (3.3) satisfies:

(F7) there exist two positive constants $\eta$ and $\beta<\varphi^{-}$such that

$$
F(t) \leq \eta\left(1+|t|^{\beta}\right) \quad \text { for } t \in \mathbb{R}
$$

(F8) there exist $0<c<k$ and $s \in \mathbb{R}$ with

$$
|\Omega| \Phi(|s|)>\left(\frac{c}{k}\right)^{\varphi^{+}}
$$

such that

$$
\sup _{|t| \leq c} F(t)<\frac{\left(\frac{c}{k}\right)^{\varphi^{+}}}{|\Omega| \Phi(|s|)} F(s)
$$

Then, setting

$$
\Lambda:=\left(\frac{\Phi(|s|)}{F(s)}, \frac{\left(\frac{c}{k}\right)^{\varphi^{+}}}{|\Omega| \sup _{|t| \leq c} F(t)}\right),
$$

for each compact interval $[a, b] \subset \Lambda$, there exists $\rho>0$ with the following property: for every $\lambda \in[a, b]$ and for every $L^{1}$-Carathéodory function $g: \Omega \times \mathbb{R} \rightarrow \mathbb{R}$, there 
exists $\delta>0$ such that, for each $\mu \in[0, \delta]$, the system (3.2) admits at least three weak solutions in $W^{1} L_{\Phi}(\Omega)$ whose norms are less than $\rho$.

As an example of our main result, we state a special case of Theorem 4.

Corollary 1. Let $f: \mathbb{R} \rightarrow \mathbb{R}$ be a non-negative (not identically zero) continuous function such that

$$
\lim _{t \rightarrow 0^{+}} \frac{f(t)}{t^{\varphi^{+}-1}}=0
$$

Assume that

$$
F(t) \leq \eta\left(1+|t|^{\beta}\right) \quad \text { for } t \in \mathbb{R}
$$

and set

$$
\Lambda:=\left(|\Omega| \inf _{s \in S} \frac{\Phi(s)}{F(s)},+\infty\right)
$$

where

$$
S:=\{s>0: F(s)>0\} .
$$

Then, for each compact interval $[a, b] \subset \Lambda$, there exists $\rho>0$ with the following property: for every $\lambda \in[a, b]$ and for every $L^{1}$-Carathéodory function $g: \Omega \times \mathbb{R} \rightarrow \mathbb{R}$, there exists $\delta>0$ such that, for each $\mu \in[0, \delta]$, the system (3.2) admits at least three weak solutions in $W^{1} L_{\Phi}(\Omega)$ whose norms are less than $\rho$.

Proof. Fix $\lambda>|\Omega| \inf _{s \in S} \frac{\Phi(s)}{F(s)}$. Then, there exists $\bar{s}$ such that $F(\bar{s})>0$ and

$$
\lambda>|\Omega| \frac{\Phi(\bar{s})}{F(\bar{s})} .
$$

From condition $\left(\ell_{0}\right)$, we have

$$
\lim _{\xi \rightarrow 0^{+}} \frac{F(\xi)}{\xi^{\varphi^{+}}}=0
$$

Therefore, we can find a positive constant $\bar{c}$ such that

$$
\bar{c}<k \min \left\{1,(|\Omega| \Phi(|s|))^{1 / \varphi^{+}}\right\},
$$

and

Hence,

$$
\frac{F(\bar{c})}{\bar{c}^{\varphi^{+}}}<\frac{1}{k^{\varphi^{+}}} \min \left\{\frac{F(\bar{s})}{|\Omega| \Phi(|s|)}, \frac{1}{\lambda|\Omega|}\right\} .
$$

$$
\lambda \in\left(\frac{\Phi(|s|)}{F(s)}, \frac{\left(\frac{c}{k}\right)^{\varphi^{+}}}{|\Omega| F(c)}\right) .
$$

The hypotheses of Theorem 4 are satisfied, so the conclusion follows.

Next, we give a concrete application of Corollary 1. 
Example 2. Let $\Omega$ be a non-empty bounded open subset of the Euclidean Space $\mathbb{R}^{N}(N \geq 3)$ with smooth boundary $\partial \Omega$, and let $\Phi$ be a Young's function that satisfies hypotheses $(\Phi 0)-(\Phi 2)$. Define $f: \mathbb{R} \rightarrow \mathbb{R}$ by

$$
f(t):= \begin{cases}0, & \text { if } t<0, \\ t^{\varphi^{+}}, & \text {if } 0 \leq t \leq 1, \\ t^{s}, & \text { if } t>1,\end{cases}
$$

where $s \in\left(0, \varphi^{-}-1\right)$. Setting

$$
\Lambda:=\left(\frac{\Phi(|s|)}{F(s)}, \frac{\left(\frac{c}{k}\right)^{\varphi^{+}}}{|\Omega| \sup _{|t| \leq c} F(t)}\right),
$$

from Corollary 1 for each compact interval $[a, b] \subset \Lambda$, there exists $\rho>0$ with the following property: for every $\lambda \in[a, b]$ and for every $L^{1}$-Carathéodory function $g$ : $\Omega \times \mathbb{R} \rightarrow \mathbb{R}$, there exists $\delta>0$ such that, for each $\mu \in[0, \delta]$, the system (3.2) admits at least three weak solutions in $W^{1} L_{\Phi}(\Omega)$ whose norms are less than $\rho$.

In particular, let $\Omega \subset \mathbb{R}^{3}$ with $|\Omega|=1$. Consider $f: \mathbb{R} \rightarrow \mathbb{R}$ defined by

$$
f(t):= \begin{cases}0, & \text { if } t<0 \\ t^{5}, & \text { if } 0 \leq t \leq 1 \\ t^{2}, & \text { if } t>1\end{cases}
$$

Set $\Phi(s):=\int_{0}^{s} \frac{t|t|^{3}}{\log (1+|t|)} d t$. Then, for every $s>0$,

$$
\frac{\Phi(s)}{F(s)}:= \begin{cases}6 \frac{\int_{0}^{s} \frac{t|t|^{3}}{\log (1+|t|)} d t}{s^{6}}, & \text { if } 0 \leq s \leq 1, \\ 6 \frac{\int_{0}^{s} \frac{t|t|^{3}}{\log (1+|t|)} d t}{2 s^{3}-1}, & \text { if } s>1 .\end{cases}
$$

Moreover, by direct computations, since the function $\frac{\Phi(s)}{F(s)}$ attains its minimum at $s_{0} \approx 1.189089126$, it follows that

$$
\inf _{s>0} \frac{\Phi(s)}{F(s)} \approx 1.804670144
$$


So, for every $L^{1}$-Carathéodory function $g: \Omega \times \mathbb{R} \rightarrow \mathbb{R}$, there exists $\delta>0$ such that, for each $\mu \in[0, \delta]$, the non-homogeneous Neumann problem

$$
\left\{\begin{array}{l}
-\operatorname{div}\left(\frac{|\nabla u|^{3}}{\log (1+|\nabla u|)} \nabla u\right)+\frac{|u|^{3}}{\log (1+|u|)} u=2 f(u)+\mu g(x, u), \quad \text { in } \quad \Omega, \\
\frac{\partial u}{\partial v}=0, \quad \text { on } \quad \partial \Omega,
\end{array}\right.
$$

admits at least three weak solutions in $W^{1} L_{\Phi}(\Omega)$.

\section{REFERENCES}

[1] R. A. Adams, Sobolev Spaces. Academic Press, New York, 1975.

[2] G. A. Afrouzi, S. Heidarkhani, and S. Shokooh, "Infinitely many solutions for Steklov problems associated to non-homogeneous differential operators through Orlicz-Sobolev spaces," Complex Var. Elliptic Equ., vol. 60, pp. 1505-1521, 2015.

[3] G. A. Afrouzi, V. Rădulescu, and S. Shokooh, "Multiple solutions of Neumann problems: an Orlicz-Sobolev space setting," Bull. Malaysian Math. Sci. Soc., to appear.

[4] G. Bonanno and S. A. Marano, "On the structure of the critical set of non-differentiable functions with a weak compactness condition," Appl. Anal., vol. 89, pp. 1-10, 2010.

[5] G. Bonanno, G. Molica Bisci, and V. Rădulescu, "Existence of three solutions for a nonhomogeneous Neumann problem through Orlicz-Sobolev spaces," Nonlinear Anal., vol. 74, pp. 4785-4795, 2011.

[6] G. Bonanno, G. Molica Bisci, and V. Rădulescu, "Arbitrarily small weak solutions for a nonlinear eigenvalue problem in Orlicz-Sobolev spaces,” Monatsh Math., vol. 165, pp. 305-318, 2012.

[7] G. Bonanno, G. Molica Bisci, and V. Rădulescu, "Quasilinear elliptic non-homogeneous Dirichlet problems through Orlicz-Sobolev spaces," Nonlinear Anal., vol. 75, pp. 4441-4456, 2012.

[8] F. Cammaroto and L. Vilasi, "Multiple solutions for a nonhomogeneous Dirichlet problem in Orlicz-Sobolev spaces," Appl. Math. Comput., vol. 218, pp. 11 518-11 527, 2012.

[9] P. Clément, B. de Pagter, G. Sweers, and F. de Thélin, "Existence of solutions to a semilinear elliptic system through Orlicz-Sobolev spaces," Mediterr. J. Math., vol. 1, pp. 241-267, 2004.

[10] P. Clément, M. García-Huidobro, R. Manásevich, and K. Schmitt, "Mountain pass type solutions for quasilinear elliptic equations," Calc. Var. Partial Differential Equations, vol. 11, pp. 33-62, 2000.

[11] G. Dankert, Sobolev Embedding Theorems in Orlicz Spaces, PhD. Thesis, University of Köln, 1966.

[12] L. Diening, "Maximal function on Musielak-Orlicz spaces and generalized Lebesgue spaces," Bull. Sci. Math., vol. 129, pp. 657-700, 2005.

[13] T. K. Donaldson, "Nonlinear elliptic boundary value problems in Orlicz-Sobolev spaces," J. Differential Equations, vol. 10, pp. 507-528, 1971.

[14] T. K. Donaldson and N. S. Trudinger, "Orlicz-Sobolev spaces and imbedding theorems," J. Functional Analysis, vol. 8, pp. 52-75, 1971.

[15] J. P. Gossez, "A strongly nonlinear elliptic problem in Orlicz-Sobolev spaces," ser. Proceedings of Symposia in Pure Mathematics, vol. 45. Amer. Math. Soc., Providence, 1986, pp. 455-462.

[16] J. R. Graef and L. Kong, Multiple Solutions of Boundary Value Problems, A Variational Approach, ser. Trends in Abstract and Applied Analysis, Vol. 1. World Scientific, 2015.

[17] N. Halidias and V. K. Le, "Multiple solutions for quasilinear elliptic Neumann problems in OrliczSobolev spaces," Bound. Value Probl., vol. 3, pp. 299-306, 2005. 
[18] A. Kristály, M. Mihăilescu, and V. Rădulescu, "Two non-trivial solutions for a non-homogeneous Neumann problem: an Orlicz-Sobolev space setting,” Proc. Roy. Soc. Edinburgh Sect. A, vol. 139, pp. 367-379, 2009.

[19] A. Kristály, V. Rădulescu, and C. Varga, "Variational principles in mathematical physics, geometry, and economics: Qualitative analysis of nonlinear equations and unilateral problems," Encyclopedia of Mathematics and its Applications, No. 136, 2010.

[20] M. Mihăilescu and V. Rădulescu, "Eigenvalue problems associated to nonhomogeneous differential operators in Orlicz-Sobolev spaces," Anal. Appl., vol. 83, pp. 235-246, 2008.

[21] M. Mihăilescu and V. Rădulescu, "Neumann problems associated to nonhomogeneous differential operators in Orlicz-Sobolev space," Ann. Inst. Fourier Grenoble, vol. 6, pp. 2087-2111, 2008.

[22] M. Mihăilescu and D. Repovš, "Multiple solutions for a nonlinear and non-homogeneous problem in Orlicz-Sobolev spaces," Appl. Math. Comput., vol. 217, pp. 6624-6632, 2011.

[23] G. Molica Bisci and V. Rădulescu, "Multiple symmetric solutions for a Neumann problem with lack of compactness," C. R. Acad. Sci. Paris, Ser. I, vol. 351, pp. 37-42, 2013.

[24] J. Musielak, Orlicz Spaces and Modular Spaces, ser. Lecture Notes in Mathematics, Vol. 1034. Springer, Berlin, 1983.

[25] J. Musielak and W. Orlicz, "On modular spaces," Studia Math., vol. 18, pp. 49-65, 1959.

[26] H. Nakano, Modulared Semi-ordered Linear Spaces. Maruzen Co. Ltd., Tokyo, 1950.

[27] R. O’Neill, "Fractional integration in Orlicz spaces," Trans. Amer. Math. Soc., vol. 115, pp. 300328, 1965.

[28] P. Pucci and J. Serrin, "Extensions of the mountain pass theorem," J. Funct. Anal., vol. 59, pp. 185-210, 1984.

[29] P. Pucci and J. Serrin, “A mountain pass theorem,” J. Differential Equations, vol. 60, pp. 142-149, 1985.

[30] M. M. Rao and Z. D. Ren, Theory of Orlicz-Spaces. Dekker, New York, 1991.

[31] B. Ricceri, "On a three critical points theorem," Arch. Math. (Basel), vol. 75, pp. 220-226, 2000.

[32] B. Ricceri, "A further refinement of a three critical points theorem," Nonlinear Anal., vol. 74, pp. 7446-7454, 2011.

[33] L. Yang, "Multiplicity of solutions for perturbed nonhomogeneous Neumann problem through Orlicz-Sobolev spaces," Abstr. Appl. Anal., vol. 2012, pp. Article ID 236 712, 10 pages, 2012.

[34] E. Zeidler, Nonlinear Functional Analysis and its Applications, Vol. II B. Springer, Berlin, 1985

Authors' addresses

Ghasem A. Afrouzi Iran

Department of Mathematics, Faculty of Mathematical Sciences, University of Mazandaran, Babolsar,

E-mail address: afrouzi@umz.ac.ir

John R. Graef

Department of Mathematics, University of Tennessee at Chattanooga, Chattanooga, TN 37403, USA

E-mail address: John-Graef@utc.edu

Saeid Shokooh

Department of Mathematics, Faculty of Sciences, Gonbad Kavous University, Gonbad Kavous, Iran

E-mail address: shokooh@gonbad.ac.ir 